\title{
Development and optimization of HPLC-MS method for simultaneous determination of four terpene trilactones in Ginkgo biloba leaf extracts based on Quality by Design principles
}

Sijie Zhang

Zhejiang University

Linlin Wu

Zhejiang University

Xiaoping Wang

Zhejiang University

Xingchu Gong

Zhejiang University

Haibin Qu ( $\square$ quhb@zju.edu.cn )

Zhejiang University https://orcid.org/0000-0002-9877-4316

\section{Research}

Keywords: Quality by design, Ginkgo biloba leaf extracts, Terpene trilactones, HPLC-MS, Hypercube design space

Posted Date: May 29th, 2020

DOI: https://doi.org/10.21203/rs.3.rs-31457/v1

License: (c) (i) This work is licensed under a Creative Commons Attribution 4.0 International License.

Read Full License 
Development and optimization of HPLC-MS method for simultaneous

determination of four terpene trilactones in Ginkgo biloba leaf extracts based on Quality by Design principles

Sijie Zhang, Linlin Wu, Xiaoping Wang, Xingchu Gong, Haibin Qu*

Pharmaceutical Informatics Institute, College of Pharmaceutical Sciences, Zhejiang University, No. 866 Yuhangtang Road, Xihu District, Hangzhou 310058, China

* Corresponding author: Prof. Haibin Qu (quhb@zju.edu.cn). Tel.: +86 571 88208428; fax: +86571 88208428. Pharmaceutical Informatics Institute, College of Pharmaceutical Sciences, Zhejiang University, No. 866 Yuhangtang Road, Xihu District, Hangzhou 310058, China. 


\section{Abstract}

Background: Ginkgo biloba leaf extract (EGBL) is one of the most commonly used and most studied herbal medicines around the world. Taking into account that previously reported HPLC-ELSD methods for terpene trilactones determination in EGBL are time-consuming with complicated sample preparation, it is reasonable and meaningful to developing a simple, sensitive and robust HPLC-MS method based on a novel analytical quality by design (AQbD) approach.

Methods: Firstly, analytical target profile (ATP) and systematic risk analysis were carried out to identify potential critical method attributes (CMAs) and critical method parameters (CMPs). Secondly, CMPs were identified using a standard partial regression coefficient method. Thirdly, Box-Behnken design (BBD) was employed to establish the quantitative relationship between CMAs and CMPs. Fourthly, the Monte Carlo simulation method was used to build hypercube design space. Then, the verification experiments were performed. Fifthly, the optimized method was validated and utilized. Finally, the paired $t$ test was used to compare the developed method with HPLC-ELSD.

Results: After the screening experiments, flow rate of mobile phase, the proportion of formic acid in the mobile phase, gas flow rate and gas temperature were identified as CMPs. Models to quantitatively describe the relationship between CMAs and CMPs were built. The operational hypercube design spaces of the HPLC-MS method for terpene trilactones analysis in EGBL were successfully calculated and found to be robust, which led to the analytical control strategy. The verification experiments were 
successfully performed within the design space and model was found to be accurate. The method had been successfully used for quality analysis of development batches of EGBL and obtained almost identical results to data determinated using HPLC.

Conclusions: In this work, an analytical control strategy for HPLC-MS method for terpene trilactones analysis in EGBL was developed using AQbD concepts, which is promising for application to other Chinese medicines. The developed HPLC-MS method is an alternative method for quantification of terpene trilactones in commercial EGBL and will be applicable throughout the life cycle of the product.

Keywords: Quality by design, Ginkgo biloba leaf extracts, Terpene trilactones, HPLC-MS, Hypercube design space

\section{Background}

Ginkgo biloba L., a "living fossil", is one of the oldest living tree species and has been existing on earth for 200 million years [1, 2]. Nowadays it is mainly present in China, Korea and Japan [2]. At the moment, the EGBL have been used in nutraceutical products due to potential benefit in alleviating symptoms associated with cognitive impairment, dementia, Alzheimer's disease [3, 4], intermittent claudication [5, 6], tinnitus [7], asthma [8] and hypertension [9].

The extracts are prepared in a multi-step process which may vary from manufacturer to manufacturer with the exact details remaining unknown. During the process some compounds are enriched while others are removed. The final extracts contain a large number of constituents from various classes. The most important 
constituents of EGBL include terpene trilactones, flavonol glycosides, biflavones, proanthocyanidins and simple phenolic acids. Currently flavonol glycosides and terpene trilactones are considered the two pharmacologically most important groups present and have gotten by far the most attention, especially terpene trilactones $[10,11]$. The content of three ginkgolides $\mathrm{A}, \mathrm{B}$ and $\mathrm{C}(\mathrm{GA}, \mathrm{GB}, \mathrm{GC})$ represent, together with the bilobalide (BB), the total terpene trilactones of EGBL in the Chinese Pharmacopoeia (2015 version). More specifically, the total terpene trilactones of EGBL is only declared to reach $6 \%$ and no information is available concerning the content of the specific ingredients. The terpene trilactones lack a strong chromophore, and it has previously been difficult to develop a suitably fast and robust LC-UV method [12]. Furthermore, tedious pre-purification procedures of the samples have been necessary in order to separate the terpene trilactones from compounds which would interfere in their detection [13-15]. In order to evaluate the distribution of these compounds in these EGBL, simple, fast and valid analytical methods are desirable.

Regarding analysis of terpene trilactones of ginkgo, separate methods are usually developed and validated. Conventional techniques such as HPLC with evaporative light scattering detection (ELSD) or refractive index (RI) detection provided a satisfactory determination of the ginkgolides and bilobalide, but these methods required a long process of sample preparation [15-17]. Gas chromatography coupled with a flame ionization detector (GC-FID) and mass spectroscopy equipped with an electron impact interface (GC-MS) have been explored, but these methods are rather time-consuming 
because derivatization of the samples is required [18, 19]. Recently, quantitative ${ }^{1} \mathrm{H}-\mathrm{NMR}$ is another method proposed for the quantification, but the signals selected to be quantified should be on a flat base-line in order to give an exact integral. This problem showed the quantification for bilobalide may still need further work [20]. In this case, HPLC-MS have become very popular in the field of quantification due to their superior sensitivity and specificity [21]. Therefore, a robust method should be developed and validated for similar cases.

Modern trends in pharmaceutical industry and regulatory documents strongly suggest the implementation of Quality by Design (QbD) concept in pharmaceutical product development, which is known as AQbD [22]. Compared to a traditional optimization of analytical method, such as the One-Factor-At-a-time approach, the AQbD approach has several obvious advantages. Firstly, it requires a small number of experiments with the understanding of critical parameters [23]. In addition, AQbD leads to the establishment of the design space, defined as the multidimensional combination and interaction of input variables that have been demonstrated to provide assurance of quality [22]. So, the obtained design space can ensure the robustness of the analysis method [24]. For this purpose, the method development starts with the establishment of the analytical target profile, which is the set of objectives of methods and define the intended purpose of the method. Meanwhile CMAs, an analogue of critical quality attributes (CQAs), are determined based on prior knowledge, such as the signal-to-noise ratio of target components, analysis time, the resolutions of critical peaks, and so on [25, 
26]. While finding CMPs is through a risk assessment and lab studies following design of experiments, just as the process of understanding critical process parameters [27]. Then the relationships between CMPs and CMAs are studied through statistical models or mechanism models. After that, the analytical design space can be established and the method quality control strategy is established from understanding of the criticality of each method parameter of each method attribute. Finally, method validation can be carried out.

In the current study, the aim was to develop and validate a simple and robust method, suitable for analysis of terpene trilactones of EGBL. Therefore, a HPLC-MS method development for the analysis of EGBL was supported by the AQbD approach. Firstly, risk assessment and a Plackett-Burman experimental design were utilized to screen the factors [25]. The relationship between the CMAs and CMPs was determined by a Box-Behnken experimental design. The analytical design space of the HPLC-MS method was performed by a Monte Carlo probability map [28]. Then the design space was verified, and method validation was carried out. Finally, the developed HPLC-MS analytical method based on AQbD was applied to determine the terpene trilactones content in EGBL and compared with the data using HPLC-ELSD. A robust and sensitive HPLC-MS method for the quantification of these four terpene trilactones in EGBL was established. Compared with the reported HPLC-ELSD methods with complicated sample preparation and long analysis time, the developed method leads to more effective. As far as our knowledge is concerned, this is the first time to establish a 
robust HPLC-MS method for simultaneous determination of four terpene trilactones in EGBL based on AQbD concept, which can improve the quality control of Ginkgo biloba leaves.

\section{Materials and Methods}

\subsection{Chemicals and reagents}

The EGBL samples were kindly provided by Jiangsu Beisikang Pharmaceutical Co. Ltd. (Pizhou, Jiangsu, China), Zhejiang conba Pharmaceutical Co. Ltd. (Hangzhou, Zhejiang, China) and Zhejiang Delekang Food Co. Ltd. (Taizhou, Zhejiang, China), respectively. The standard substances of bilobalide, ginkgolide A, ginkgolide B, and ginkgolide C (98\%) were purchased from Shanghai Winherb Medical Technology Co Co., Ltd (Shanghai, China). HPLC-grade methanol and formic acid were supplied by Merck (Darmstadt, Germany). Ultrapure water was produced in the laboratory with a Milli-Q water purification system (Miford, MA, USA). All of the other reagents and chemicals were of analytical grade (Sinopharm Chemical Reagent Co., Shanghai, China).

All the standard substances were dissolved in $50 \%$ methanol/water (v/v) and stored at $4{ }^{\circ} \mathrm{C}$ prior to analysis.

\subsection{Samples preparation}

The $3 \mathrm{mg}$ EGBL of each batch was extracted with $10 \mathrm{~mL} \mathrm{50 \%} \mathrm{methanol} \mathrm{aqueous}$ solution for $5 \mathrm{~min}$ in an ultrasonic water bath (KQ-250 ultrasonic cleaner, Kunshan Ultrasonic instruments CO., LTD, China). After extraction, the weight loss was 
complemented with $50 \%$ methanol aqueous solution. After that, each sample solution was filtered through a $0.22 \mu \mathrm{m}$ syringe filter before HPLC-MS analysis. All samples were analyzed in triplicates.

\subsection{HPLC-MS analysis}

The quantitative analysis of the terpene trilactones was carried out using an LC-MS system consisted of Agilent 1100 series LC system (Agilent Technologies, Waldbronn, Germany), composed of degasser, quaternary pump, thermostated autosampler coupled with mass spectrometer, 1946D Series Single Quadrupole from Agilent Technologies equipped with an ESI source. Data acquisition was carried out in single ion monitoring mode with negative polarity ionization and the quantitative ions for $\mathrm{BB}(\mathrm{m} / \mathrm{z} 325)$, GC $(\mathrm{m} / \mathrm{z} 439)$ and GB $(\mathrm{m} / \mathrm{z} 423)$ were all their specific deprotonated molecules $[\mathrm{M}-\mathrm{H}]^{-}$, while GA (m/z 453) was [M-H+COOH]-. FIA from Agilent Technologies was used for the individual values selection of the fragmentor for each compound.

The chromatographic separation was performed on a reversed phase Zorbax SB-C18 column $(4.6 \times 250 \mathrm{~mm}, 5 \mu \mathrm{m})$ from Agilent Technologies. The samples and standards were separated with linear gradient elution. The mobile phase was composed of solvent A (an appropriate amount of formic acid in water) and solvent B (methanol). A mixed standard stock solution containing $\mathrm{BB}, \mathrm{GC}, \mathrm{GA}$ and $\mathrm{GB}$ was prepared. Standard solutions with other concentrations were prepared by diluting the stock solution with $50 \%(\mathrm{v} / \mathrm{v})$ aqueous methanol. All standards were filtered through a 0.22 $\mu \mathrm{m}$ syringe filter and subjected to HPLC analysis. The gradient profile as follows: $0-10$ 
$\min , 20 \%-50 \% \mathrm{~B} ; 10-20 \mathrm{~min}, 50 \%-80 \% \mathrm{~B}$. In addition, there was a column wash of $100 \% \mathrm{~B}$ in mobile phase for $7 \mathrm{~min}$ after each run and column equilibration with initial mobile phase composition for $7 \mathrm{~min}$. The injection volume for all samples or standards was $10 \mu \mathrm{L}$. Calibration curves were established, then the quantitative analyses of samples were based on the calibration plots of the selected ion peak areas versus the concentrations for $\mathrm{BB}, \mathrm{GC}, \mathrm{GA}$ and $\mathrm{GB}$, respectively.

\subsection{Experimental design}

In accordance with the experimental design, some factors were investigated, including the flow rate of the mobile phase $\left(X_{1}\right)$, the proportion of formic acid in the mobile phase $\left(X_{2}\right)$, column temperature $\left(X_{3}\right)$, gas flow rate $\left(X_{4}\right)$, gas temperature $\left(X_{5}\right)$, nebulizer pressure $\left(X_{6}\right)$, and capillary voltage $\left(X_{7}\right)$. The coded and uncoded values of each parameter are shown in Table 1. And then the effects of these seven parameters on the analytical responses were performed by a Plackett-Burman experimental design with three center points, as shown in Table 2.

Table 1 The factors and levels of Plackett-Burman designed experiments

\begin{tabular}{lcccccc}
\hline \multicolumn{1}{c}{ Factor } & \multirow{2}{*}{ Term } & Unit & \multicolumn{3}{c}{ Code } \\
\cline { 5 - 7 } & & & -1 & 0 & 0.7 \\
\hline The flow rate of mobile phase & $X_{1}$ & $\mathrm{~mL} / \mathrm{min}$ & 0.5 & 0.6 & 0.1 \\
The proportion of formic acid in the mobile phase & $X_{2}$ & $\%$ & 0.050 & 0.075 & 0.100 \\
Column temperature & $X_{3}$ & ${ }^{\circ} \mathrm{C}$ & 20 & 30 & 40 \\
Gas flow rate & $X_{4}$ & $\mathrm{~L} / \mathrm{min}$ & 8 & 10 & 12 \\
Gas temperature & $X_{5}$ & ${ }^{\circ} \mathrm{C}$ & 300 & 325 & 350 \\
Nebulizer pressure & $X_{6}$ & $\mathrm{psi}$ & 20 & 35 & 50 \\
Capillary voltage & $X_{7}$ & $\mathrm{v}$ & 3000 & 4000 & 5000 \\
\hline
\end{tabular}


Table 2 The conditions and results of Plackett-Burman designed experiments

\begin{tabular}{cccccccccccc}
\hline \multirow{2}{*}{ Run } & \multicolumn{1}{c}{ Analytical parameters } & \multicolumn{7}{c}{ CMAs } \\
\cline { 2 - 12 } & $X_{1}$ & $X_{2}$ & $X_{3}$ & $X_{4}$ & $X_{5}$ & $X_{6}$ & $X_{7}$ & $Y_{1}$ & $Y_{2}$ & $Y_{3}$ & $Y_{4}$ \\
\hline 1 & 0.6 & 0.075 & 30 & 10 & 325 & 35 & 4000 & 6.46 & 6.00 & 6.26 & 6.13 \\
2 & 0.7 & 0.050 & 40 & 12 & 300 & 50 & 5000 & 6.32 & 5.92 & 6.10 & 6.02 \\
3 & 0.6 & 0.075 & 30 & 10 & 325 & 35 & 4000 & 6.47 & 6.00 & 6.27 & 6.14 \\
4 & 0.5 & 0.100 & 40 & 8 & 350 & 50 & 5000 & 6.40 & 5.97 & 6.26 & 6.15 \\
5 & 0.7 & 0.050 & 20 & 8 & 350 & 20 & 5000 & 6.37 & 5.89 & 6.19 & 6.08 \\
6 & 0.5 & 0.050 & 20 & 12 & 300 & 50 & 5000 & 6.55 & 6.10 & 6.34 & 6.23 \\
7 & 0.7 & 0.100 & 20 & 12 & 350 & 50 & 3000 & 6.39 & 5.96 & 6.16 & 6.12 \\
8 & 0.5 & 0.050 & 40 & 8 & 350 & 50 & 3000 & 6.45 & 5.99 & 6.13 & 6.07 \\
9 & 0.6 & 0.075 & 30 & 10 & 325 & 35 & 4000 & 6.47 & 6.01 & 6.27 & 6.14 \\
10 & 0.7 & 0.100 & 20 & 8 & 300 & 50 & 3000 & 6.16 & 5.75 & 5.97 & 5.85 \\
11 & 0.5 & 0.100 & 20 & 12 & 350 & 20 & 5000 & 6.49 & 6.05 & 6.35 & 6.24 \\
12 & 0.5 & 0.050 & 20 & 8 & 300 & 20 & 3000 & 6.46 & 5.98 & 6.28 & 6.12 \\
13 & 0.5 & 0.100 & 40 & 12 & 300 & 20 & 3000 & 6.44 & 5.99 & 6.23 & 6.14 \\
14 & 0.7 & 0.10 & 40 & 8 & 300 & 20 & 5000 & 6.19 & 5.67 & 6.09 & 5.81 \\
15 & 0.7 & 0.050 & 40 & 12 & 350 & 20 & 3000 & 6.45 & 6.00 & 6.15 & 6.15 \\
\hline
\end{tabular}

After screening experiments, CMPs were fixed and other parameters were kept constant. The fixed parameters as follows: column temperature of $30{ }^{\circ} \mathrm{C}$, nebulizer pressure of $35 \mathrm{psi}$, capillary voltage of $3500 \mathrm{v}$. Then the quantitative relationships between the CMPs and the CMAs were carried out using a BBD with three center points, as shown in Table 3.

Table 3 The conditions and results of the Box-Behnken designed experiments

\begin{tabular}{ccccccccc}
\hline \multirow{2}{*}{ Run } & \multicolumn{3}{c}{ CMPs } & \multicolumn{5}{c}{ CMAs } \\
\cline { 2 - 9 } & $\mathrm{X}_{1}(\mathrm{~mL} / \mathrm{min})$ & $\mathrm{X}_{2}(\%)$ & $\mathrm{X}_{4}(\mathrm{~L} / \mathrm{min})$ & $\mathrm{X}_{5}\left({ }^{\circ} \mathrm{C}\right)$ & $\operatorname{logBB}$ & $\log \mathrm{C}$ & $\log \mathrm{gB}$ & $\log \mathrm{AA}$ \\
\hline 1 & 0.6 & 0.100 & 10 & 350 & 6.21 & 5.70 & 5.93 & 5.85 \\
2 & 0.6 & 0.075 & 8 & 350 & 6.20 & 5.70 & 5.95 & 5.85 \\
3 & 0.6 & 0.050 & 8 & 325 & 6.17 & 5.72 & 5.96 & 5.87 \\
4 & 0.5 & 0.075 & 12 & 325 & 6.31 & 5.80 & 6.02 & 5.90 \\
5 & 0.7 & 0.075 & 10 & 350 & 6.21 & 5.71 & 5.96 & 5.88 \\
6 & 0.5 & 0.075 & 8 & 325 & 6.20 & 5.73 & 5.98 & 5.84
\end{tabular}




\begin{tabular}{ccccccccc}
7 & 0.6 & 0.075 & 10 & 325 & 6.22 & 5.73 & 5.97 & 5.88 \\
8 & 0.7 & 0.075 & 12 & 325 & 6.20 & 5.71 & 5.95 & 5.88 \\
9 & 0.6 & 0.050 & 10 & 300 & 6.18 & 5.75 & 5.99 & 5.90 \\
10 & 0.6 & 0.050 & 10 & 350 & 6.31 & 5.82 & 6.04 & 5.96 \\
11 & 0.6 & 0.075 & 10 & 325 & 6.22 & 5.73 & 5.97 & 5.88 \\
12 & 0.5 & 0.075 & 10 & 350 & 6.32 & 5.82 & 6.04 & 5.92 \\
13 & 0.7 & 0.075 & 8 & 325 & 6.07 & 5.60 & 5.85 & 5.77 \\
14 & 0.7 & 0.100 & 10 & 325 & 6.12 & 5.62 & 5.86 & 5.79 \\
15 & 0.5 & 0.075 & 10 & 300 & 6.22 & 5.76 & 5.99 & 5.85 \\
16 & 0.6 & 0.100 & 8 & 325 & 6.08 & 5.61 & 5.86 & 5.74 \\
17 & 0.5 & 0.100 & 10 & 325 & 6.24 & 5.74 & 5.96 & 5.84 \\
18 & 0.6 & 0.100 & 10 & 300 & 6.08 & 5.63 & 5.88 & 5.76 \\
19 & 0.5 & 0.050 & 10 & 325 & 6.34 & 5.87 & 6.07 & 5.96 \\
20 & 0.6 & 0.075 & 12 & 300 & 6.19 & 5.72 & 5.95 & 5.85 \\
21 & 0.7 & 0.050 & 10 & 325 & 6.21 & 5.74 & 5.99 & 5.91 \\
22 & 0.7 & 0.075 & 10 & 300 & 6.08 & 5.63 & 5.9 & 5.80 \\
23 & 0.6 & 0.075 & 8 & 300 & 6.06 & 5.58 & 5.87 & 5.70 \\
24 & 0.6 & 0.100 & 12 & 325 & 6.20 & 5.70 & 5.91 & 5.83 \\
25 & 0.6 & 0.075 & 10 & 325 & 6.22 & 5.73 & 5.97 & 5.88 \\
26 & 0.6 & 0.050 & 12 & 325 & 6.30 & 5.81 & 6.03 & 5.94 \\
27 & 0.6 & 0.075 & 12 & 350 & 6.27 & 5.78 & 6.00 & 5.92 \\
\hline
\end{tabular}

\subsection{Data processing}

To estimate which parameters were significant for response values, the standard partial regression coefficient method was used to evaluate the results of Plackett-Burman experimental design and select CMPs [29]. Firstly, the response values were standardized according to Eq (1).

$$
Y_{k}^{\prime}=\frac{Y_{k}-\bar{Y}_{k}}{S D_{k}}(k=1,2,3,4)
$$

Where $Y_{k}, \bar{Y}_{k}$, and $Y_{k}^{\prime}$ represent the measured value, the average values of each response and the standardized value respectively; $S D_{k}$ is the standard deviation of each response; and number $\mathrm{k}(\mathrm{k}=1,2,3,4)$ represents ion peak areas of the $\mathrm{BB}, \mathrm{GC}, \mathrm{GA}$ and GB, respectively. Then, multiple linear regression analysis was used to calculate 
standard partial regression coefficients according to $\mathrm{Eq}(2)$.

$$
Y_{k}^{\prime}=a_{0, \mathrm{k}}+\sum_{i=1}^{7} a_{i, k} X_{i}(i=1,2, \ldots, 7)
$$

Where $a_{0, \mathrm{k}}$ is a constant; $X_{i}$ is potential CMPs; and $a_{i, k}$ represents the standard partial regression coefficient. The absolute values of each $a_{i, k}$ were summed up to analyze the total influences of each parameter $X_{i}$ on all the responses, as seen in Eq (3).

$$
A_{i}=\sum_{k=1}^{4}\left|a_{i, k}\right|
$$

Where $A_{i}$ is named as importance factor. Parameters with higher $A_{i}$ values were expected to have greater influences on the responses.

In the Box-Behnken designed experiments, quadratic models which can be expressed as Eq (4) were built to analyze the relationships between CMPs and CMAs.

$$
Y=b_{0}+\sum_{i=1}^{k} b_{i} X_{i}+\sum_{i=1}^{k} b_{i i} \mathrm{X}_{\mathrm{i}}^{2}+\sum_{i>j}^{k} b_{i j} X_{i} X_{j}
$$

Where $b_{0}$ is a constant, $b_{i}, b_{i i}$ and $b_{i j}$ are regression coefficients, $Y$ indicates the response, and $X_{i}$ and $X_{j}$ are the independent variables. The analysis of the results was performed using Design Expert (version 11.0.5, Stat-Ease Inc., USA). Analysis of variance and relative statistical analysis was carried out. The statistical analysis was based on a confidence level of 0.90 , and insignificant terms were removed using a stepwise regression.

Recently, probability-based design space has come to the forefront because it can make the method robust. Based on the special goals of CMAs, a Monte-Carlo method was performed using MODDE Pro (version 12.0.1, Umetrics, Sweden) to calculate the design space. The limit of failure probability was set at $1 \%$ with 50000 times iterations. 
The model error was included, and the simulation resolution was set at 16 .

\subsection{Method validation}

After the design space was obtained, the linearity, sensitivity, analytical precision, accuracy, stability and robustness experiments of developed method were carried out. The robustness testing was performed using a Plackett-Burman design experiment. The limit of detection (LOD) and the limit of quantification (LOQ) were assessed basing on an $\mathrm{S} / \mathrm{N}$ at 3:1 and 10:1, respectively. The same standard solution was injected for six times continuously to evaluate injection precision. For evaluation of intra-day precision, six sample solutions were prepared in parallel and analyzed during a single day. For evaluation of inter-day precision, replicate samples were analyzed for six consecutive days, respectively. The stability studies were performed for $48 \mathrm{~h}$, including measurements at $0,2,4,6,8,10,12,24,48 \mathrm{~h}$. For evaluation of method accuracy, recovery experiments conducted in triplicate at concentration levels of 80, 100, and 120 percent of the sample solution. All results were evaluated by RSD values of the peak areas or the contents of corresponding components.

\section{Results}

\subsection{CMAs and CMPs identification}

Typical selected ion monitoring (SIM) chromatograms of EGBL and standard solution are shown in Fig.1. The goal of the current optimization procedure was to maximize analyte responses. Therefore, the selected ion peak areas of the four terpene lactones considered to be CMAs. The index values of CMAs were collected in the 
Plackett-Burman design experiment, as shown in Table 2.
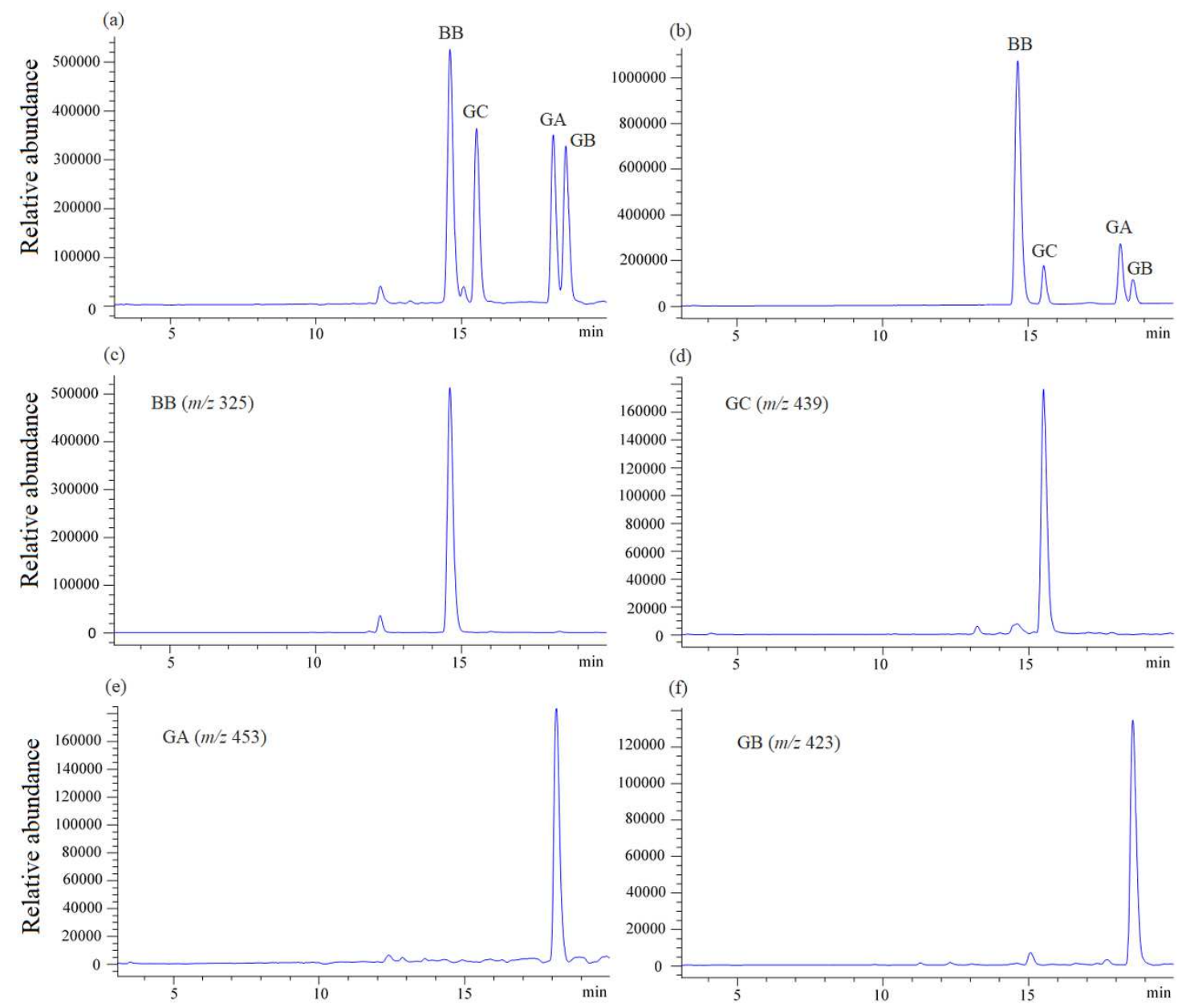

Fig. 1 Typical selected ion monitoring (SIM) chromatograms of EGBL and standard solution. (a-b) All terpene trilactones ions traces for EGBL and standard solution, respectively; (c-f) The SIM spectra of BB, GC, GA, GB for EGBL, respectively

As we all known, a lot of potential factors may affect the results of a HPLC-MS method. An Ishikawa diagram analysis was performed to obtain an initial list of factors that influence the process CMAs. As shown in Fig.2, five main causes were involved, including environment, sample injection, LC condition, ESI ion source and data 
analysis. The risk of the potential factors was determined based on prior knowledge in this work. The flow rate of mobile phase, the proportion of formic acid in the mobile phase, column temperature, gas temperature, gas flow rate, nebulizer pressure and capillary voltage were considered to be potential CMPs.

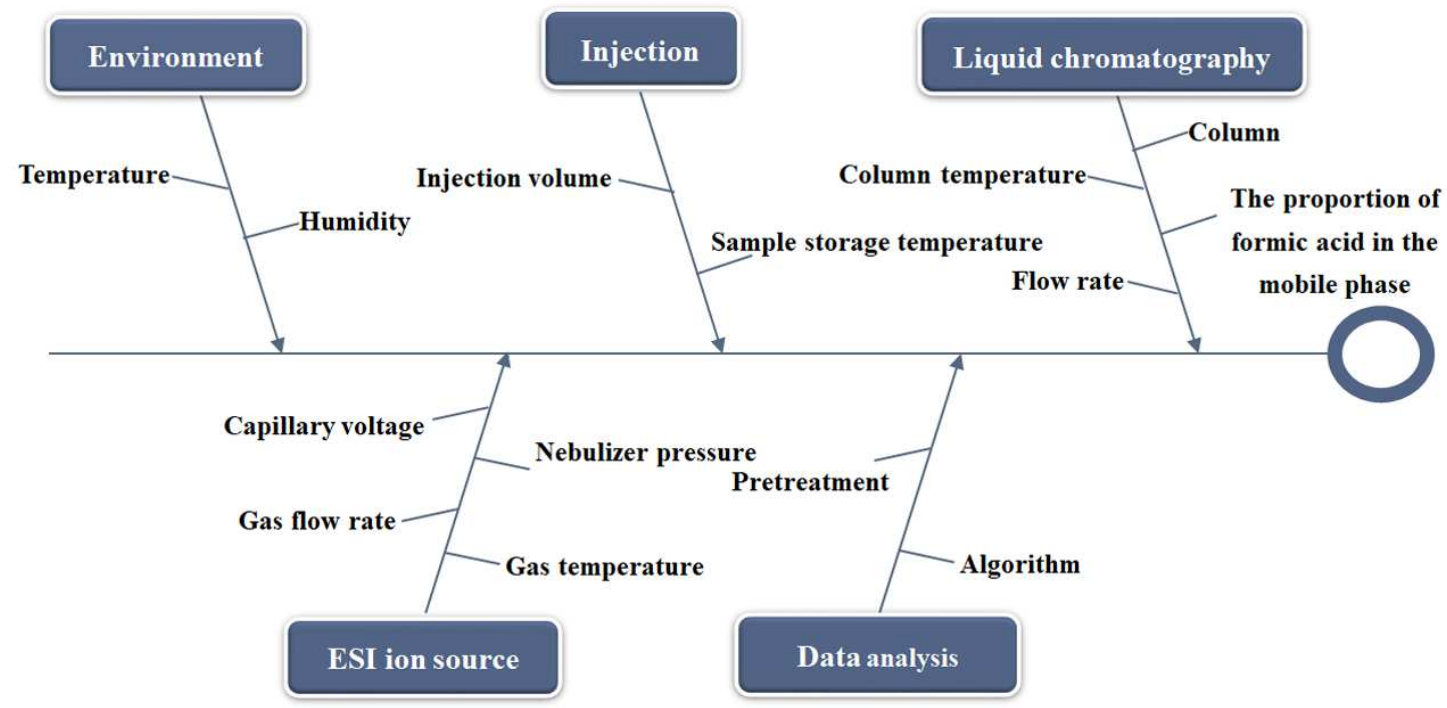

Fig. 2 Ishikawa diagram for potential CMPs selection

The results of multiple linear regression analysis and the normalized regression coefficients of each response are shown in Table 4 . The analysis parameters with the higher $A_{\mathrm{i}}$ value in the top four of all the seven potential CMPs were selected as CMPs. It was concluded that the flow rate of mobile phase, the proportion of formic acid in the mobile phase, gas flow rate and gas temperature were CMPs. The other parameters were kept constant as follows. Column temperature was $30{ }^{\circ} \mathrm{C}$ and nebulizer pressure was 35 psi. Capillary voltage was $3500 \mathrm{v}$. 
Table 4 Results of the multiple linear regression analysis

\begin{tabular}{cccccc}
\hline Parameters & $Y_{1}$ & $Y_{2}$ & $Y_{3}$ & $Y_{4}$ & $\mathrm{~A}_{\mathrm{i}}$ \\
\hline$X_{1}$ & -0.7 & -0.67 & -0.75 & -0.64 & 2.760 \\
$X_{2}$ & -0.4 & -0.38 & -0.11 & -0.26 & 1.150 \\
$X_{3}$ & -0.12 & -0.15 & -0.27 & -0.2 & 0.740 \\
$X_{4}$ & 0.48 & 0.56 & 0.34 & 0.57 & 1.950 \\
$X_{5}$ & 0.34 & 0.34 & 0.19 & 0.44 & 1.310 \\
$X_{6}$ & -0.1 & 0.088 & -0.27 & -0.071 & 0.529 \\
$X_{7}$ & -0.019 & -0.058 & 0.32 & 0.056 & 0.453 \\
\hline
\end{tabular}

\subsection{Optimization of fragmentor voltages and sample preparation conditions}

In order to obtain the main quantitative ions of each compound, the fragmentor voltages were optimized by FIA, as shown in Additional file 1: Table S1. The optimized fragmentor voltages for $\mathrm{BB}, \mathrm{GC}, \mathrm{GA}$, and GB were as follows: 80,100, 80 and $80 \mathrm{eV}$, respectively. Meanwhile, as shown in Fig.3, compound BB exhibited only a main characteristic product ion $\mathrm{m} / \mathrm{z} 325[\mathrm{M}-\mathrm{H}]^{-}$for quantitative determination. For the other three compounds, we found that the proportion of main characteristic ions of the certain concentration of samples and standard solution was almost identical. Therefore, the main characteristic product ions at $\mathrm{m} / \mathrm{z} 439, \mathrm{~m} / \mathrm{z} 453$ and $\mathrm{m} / \mathrm{z} 423$ were extracted for quantitative analysis of GC, GA, GB, respectively. In addition, the extraction time and methanol concentration were optimized by One-Factor-At-a-time approach, as shown in Additional file 1: Table S2. Firstly, different extraction time including $2 \mathrm{~min}, 5 \mathrm{~min}$ and 10 min were compared. Then the extraction time was chosen as 5 min based on extraction efficiency and time saving. Secondly, the solvents were consisted of $50 \%$, $80 \%$, and $100 \%$ methanol, respectively, and methanol concentrations were compared according to peak area of target components. Considering the solvent saving and 
extraction efficiency, $50 \%$ methanol was selected.
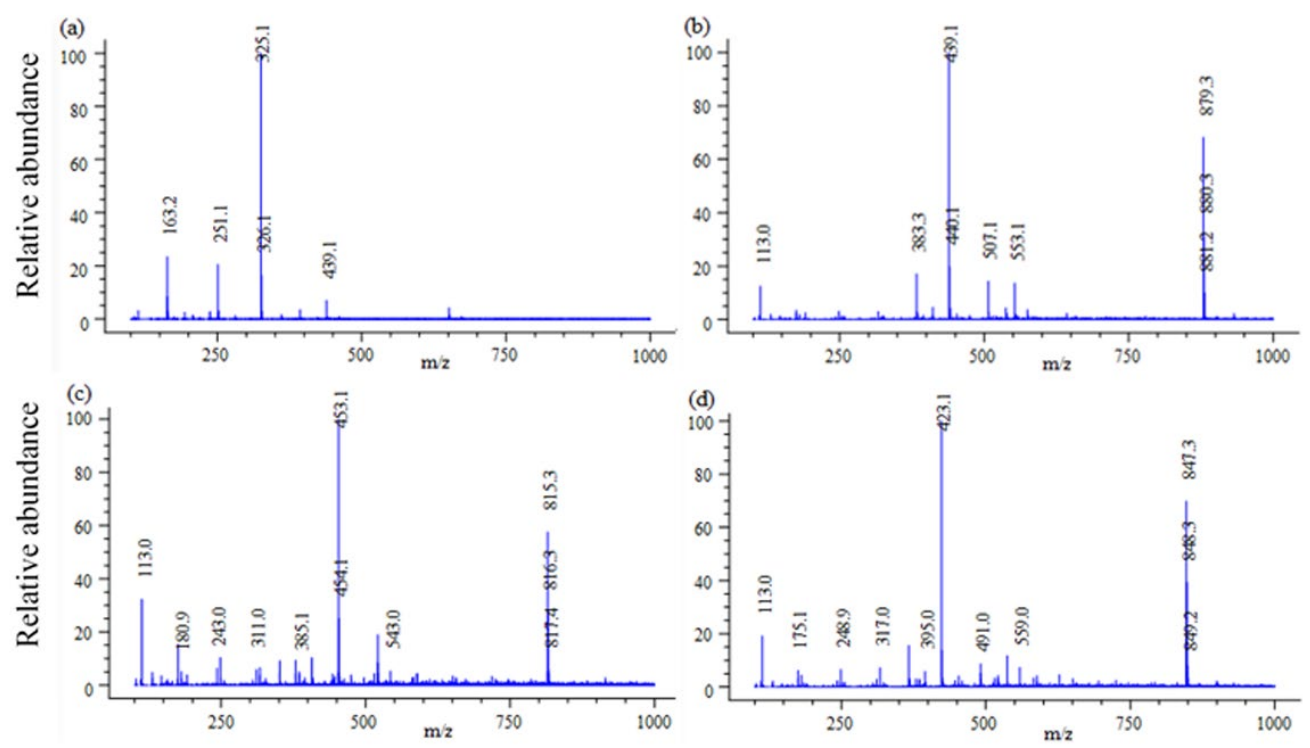

Fig. 3 Full scan mass spectrum of (a) Bilobalide; (b) Ginkgolide C; (c) Ginkgolide A; (d) Ginkgolide B.

\subsection{Effects of CMPs on CMAs}

The experimental results of Box-Behnken experiments are shown in Table 3. Table 5 presents the results including the estimated regression coefficients, the analysis of variance of all models and the $p$-values of the parameters. For each CMA, $R^{2}$ value was more than 0.98 indicates that most variations of experimental data can be explained. According to $p$-values of each model term, the linear effects of four CMPs were significant for all four CMAs. The interaction term between gas flow rate and gas temperature was significant for all four CMAs, while flow rate of mobile phase and gas flow rate for GC, GA and GB. The contour plots were obtained to analyze the effects of CMPs on each CMA. As shown in Additional file 1: Fig. S1, 2, 3, and 4, a low flow rate 
of mobile phase would lead to a high response for all four CMAs. Higher gas flow rate and gas temperature are beneficial to guarantee higher response values for all four CMAs for the range of values tested in the study.

Table 5 ANOVA results for multiple regression models

\begin{tabular}{|c|c|c|c|c|c|c|c|c|}
\hline \multirow{2}{*}{$\begin{array}{c}\text { Model } \\
\text { terms }\end{array}$} & \multicolumn{2}{|c|}{$\log B B$} & \multicolumn{2}{|c|}{$\log G C$} & \multicolumn{2}{|c|}{$\log \mathrm{GA}$} & \multicolumn{2}{|c|}{$\log G B$} \\
\hline & Coefficient & $p$ value & Coefficient & $p$ value & Coefficient & $p$ value & Coefficient & $p$ value \\
\hline Intercept & 6.21 & - & 5.74 & - & 5.98 & - & 5.88 & - \\
\hline$X_{1}$ & -0.061 & $<0.0001$ & -0.0605 & $<0.0001$ & -0.0455 & $<0.0001$ & -0.024 & $<0.0001$ \\
\hline$X_{2}$ & -0.047 & $<0.0001$ & -0.0590 & $<0.0001$ & -0.0583 & $<0.0001$ & -0.061 & $<0.0001$ \\
\hline$X_{4}$ & 0.058 & $<0.0001$ & 0.0484 & $<0.0001$ & 0.0325 & $<0.0001$ & 0.0458 & $<0.0001$ \\
\hline$X_{5}$ & 0.059 & $<0.0001$ & 0.0398 & $<0.0001$ & 0.0265 & $<0.0001$ & 0.0428 & $<0.0001$ \\
\hline$X_{1} X_{4}$ & - & - & 0.0084 & 0.0269 & 0.0136 & 0.0001 & 0.0115 & 0.0214 \\
\hline$X_{1} X_{5}$ & 0.0084 & 0.0489 & - & - & - & - & - & - \\
\hline$X_{4} X_{5}$ & -0.016 & 0.0008 & -0.0135 & 0.0011 & -0.0064 & 0.0321 & -0.0211 & 0.0002 \\
\hline$X_{I}^{2}$ & 0.0074 & 0.0371 & - & - & & & - & - \\
\hline$X_{2}^{2}$ & - & - & - & - & -0.0077 & 0.0034 & - & - \\
\hline$X_{4}^{2}$ & -0.0270 & $<0.0001$ & -0.0280 & $<0.0001$ & -0.0270 & $<0.0001$ & -0.0298 & $<0.0001$ \\
\hline$X_{5}^{2}$ & -0.0130 & 0.0007 & -0.0110 & 0.0008 & -0.0065 & 0.0104 & -0.0140 & 0.0011 \\
\hline$R^{2}$ & \multicolumn{2}{|c|}{0.9934} & \multicolumn{2}{|c|}{0.9937} & \multicolumn{2}{|c|}{0.9944} & \multicolumn{2}{|c|}{0.9862} \\
\hline$P$ value & \multicolumn{2}{|c|}{$<0.0001$} & \multicolumn{2}{|c|}{$<0.0001$} & \multicolumn{2}{|c|}{$<0.0001$} & \multicolumn{2}{|c|}{$<0.0001$} \\
\hline
\end{tabular}

Only $\mathrm{p}<0.1$ were listed

\subsection{Design space development and verification}

To obtain optimum analysis results, the design space were calculated based on the specific goals of each CMA. According to the preliminary experiment, when $\operatorname{logBB}$ was greater than $6.20, \log \mathrm{GC}$ greater than 5.70, $\log \mathrm{GA}$ greater than 5.92, $\log \mathrm{GB}$ greater than 5.85, the analytical method has good quantitative analysis effect. Therefore, the minimum values of four CMAs, $\log B B, \log G C, \log G A$ and $\log G B$ were set to 6.20 , 5.70, 5.92 and 5.85, respectively. Then, the Monte-Carlo method was performed to 
calculate the design space with a $1 \%$ probability to fall outside the specification limits. The design space obtained were irregular in shape, as shown in Fig.4 (a)-4 (c). The green areas are part of the design space with a $1 \%$ probability to fall outside the specification limits. The risk of error increases gradually from yellow to red. To minimise the chances of method failure, the largest possible hypercube design space was calculated, within the design space. The operational hypercube in design space is depicted as a gray dashed frame in Fig.4. The setpoint, which is a robust or best compromise condition, was also explored and displayed as arrow crossing. The hypercube design space and setpoint values are listed in Table 6. Then verification experiments were carried out with the robust setpoint and edge points in design space. The experimental and predicted results are shown in Table 7. These results indicate that the target specification can be achieved by conditions within the design space even at the edge point. The experimental values of four CMAs were roughly consistent with the predicted values, which may be related to the higher $R^{2}$ value of the models used for prediction. 
(a)
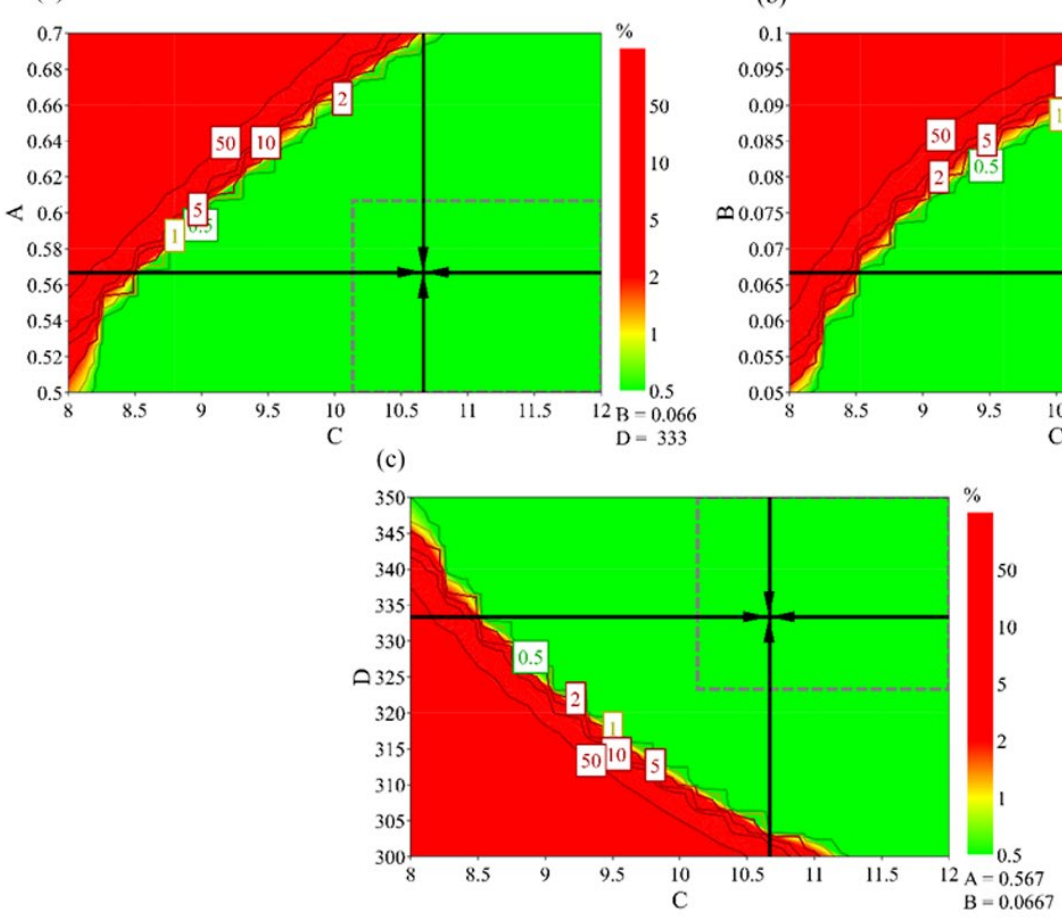

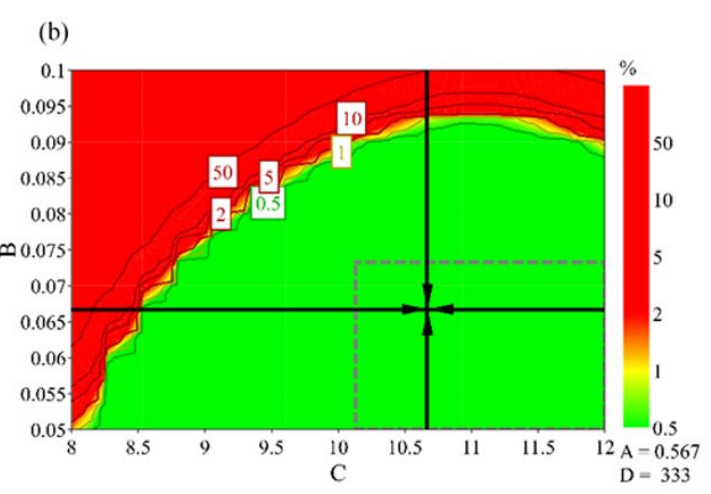

Fig. 4 Design space plot with a robust setpoint. A to D represent flow rate of mobile phase, the proportion of formic acid in the mobile phase, gas flow rate and gas temperature, respectively. (Color bar refers to the risk of error to attain CMA goals).

The largest hypercube design space is plotted as a gray dashed frame with a probability of failure of less than $1 \%$. The robust setpoint is displayed as arrow crossing.

Table 6 Design space and setpoint of parameters

\begin{tabular}{lccc}
\hline Parameters & Study range & Design space & Robust setpoint \\
\hline Flow rate of mobile phase $(\mathrm{mL} / \mathrm{min})$ & $0.5-0.7$ & $0.500-0.607$ & 0.567 \\
The proportion of formic acid in the mobile phase $(\%)$ & $0.05-0.10$ & $0.050-0.073$ & 0.067 \\
Gas flow rate $(\mathrm{L} / \mathrm{min})$ & $8-12$ & $10.1-12.0$ & 10.7 \\
Gas temperature $\left({ }^{\circ} \mathrm{C}\right)$ & $300-350$ & $323.3-350.0$ & 333.3 \\
\hline
\end{tabular}


Table7 Verification conditions and results

\begin{tabular}{llll}
\hline Verification condition & $\mathrm{V} 1$ & $\mathrm{~V} 2$ & $\mathrm{~V} 3$ \\
\hline Flow rate of mobile phase $(\mathrm{mL} / \mathrm{min})$ & 0.567 & 0.600 & 0.600 \\
The proportion of formic acid in the mobile phase $(\%)$ & 0.067 & 0.050 & 0.054 \\
Gas flow rate $(\mathrm{L} / \mathrm{min})$ & 10.7 & 10.1 & 10.3 \\
Gas temperature $\left({ }^{\circ} \mathrm{C}\right)$ & 333.3 & 350.0 & 350.0 \\
Experimental value of $\operatorname{logBB}$ & 6.52 & 6.53 & 6.50 \\
Predicted value of $\operatorname{logBB}$ & 6.29 & 6.31 & 6.30 \\
Experimental value of $\log \mathrm{GC}$ & 6.07 & 6.05 & 6.01 \\
Predicted value of $\log \mathrm{GC}$ & 5.81 & 5.83 & 5.82 \\
Experimental value of $\log \mathrm{GA}$ & 6.23 & 6.22 & 6.18 \\
Predicted value of $\log \mathrm{GA}$ & 6.04 & 6.05 & 6.04 \\
Experimental value of $\log \mathrm{GB}$ & 6.13 & 6.13 & 6.09 \\
Predicted value of $\log \mathrm{GB}$ & 5.94 & 5.97 & 5.96 \\
\hline
\end{tabular}

\subsection{Method verification}

Validation is an essential prerequisite in the development of a robust, reliable and reproducible analytical method. The robustness of an analysis method has been defined as being the capacity to remain unaffected by small but deliberately introduced variations in method parameters [30]. In order to evaluate the effects of simultaneous minor changes of the experimental conditions on the CMAs, a Plackett-Burman design experiment was carried out to analyze whether the analytical results could meet the expected requirements when the minor deviations of operating point at the edge of the design space appeared. As shown in Table 8, the analytical results of all experiment conditions have met the requirements. These results demonstrate that the developed HPLC-MS method is robust against small changes in the experimental conditions. 
Table 8 Experimental design to test the robustness of the developed method within the design space

\begin{tabular}{cccccccccccc}
\hline Run & $X_{1}$ & $X_{2}$ & $X_{3}$ & $X_{4}$ & $X_{5}$ & $X_{6}$ & $X_{7}$ & $\operatorname{logBB}$ & $\log \mathrm{GC}$ & $\operatorname{logGA}$ & $\log \mathrm{GB}$ \\
\hline 1 & 0.500 & 0.050 & 32 & 10.1 & 350.0 & 37 & 3450 & 6.37 & 5.89 & 6.10 & 5.96 \\
2 & 0.500 & 0.073 & 32 & 10.1 & 350.0 & 37 & 3550 & 6.34 & 5.84 & 6.05 & 5.92 \\
3 & 0.607 & 0.050 & 32 & 12.0 & 323.3 & 37 & 3550 & 6.48 & 5.98 & 6.17 & 6.04 \\
4 & 0.500 & 0.073 & 32 & 12.0 & 323.3 & 33 & 3450 & 6.40 & 5.91 & 6.11 & 6.96 \\
5 & 0.607 & 0.073 & 32 & 10.1 & 323.3 & 33 & 3550 & 6.29 & 5.83 & 6.07 & 5.92 \\
6 & 0.500 & 0.050 & 28 & 10.1 & 323.3 & 33 & 3450 & 6.38 & 5.89 & 6.15 & 6.02 \\
7 & 0.607 & 0.073 & 28 & 10.1 & 323.3 & 37 & 3450 & 6.28 & 5.82 & 6.04 & 5.94 \\
8 & 0.500 & 0.073 & 28 & 12.0 & 350.0 & 33 & 3550 & 6.40 & 5.90 & 6.11 & 5.99 \\
9 & 0.607 & 0.073 & 28 & 12.0 & 350.0 & 37 & 3450 & 6.35 & 5.87 & 6.09 & 5.98 \\
10 & 0.500 & 0.050 & 28 & 12.0 & 323.3 & 37 & 3550 & 6.45 & 5.96 & 6.15 & 6.07 \\
11 & 0.607 & 0.050 & 32 & 12.0 & 350.0 & 33 & 3450 & 6.41 & 5.94 & 6.15 & 6.02 \\
12 & 0.607 & 0.050 & 28 & 10.1 & 350.0 & 33 & 3550 & 6.36 & 5.88 & 6.11 & 5.99 \\
\hline
\end{tabular}

Method validation experiments were performed using the analytical conditions of method V2. The operating conditions were shown as follows. The flow rate of mobile phase was $0.6 \mathrm{~mL} / \mathrm{min}$. The proportion of formic acid in the mobile phase was $0.05 \%$. Column temperature was $30{ }^{\circ} \mathrm{C}$. Gas temperature was $350{ }^{\circ} \mathrm{C}$. Gas flow rate was 10.1 $\mathrm{L} / \mathrm{min}$. Nebulizer pressure was 35 psi and capillary voltage was $3500 \mathrm{v}$.

The linearity of the analysis method was confirmed by establishing calibration curves for four terpene trilactones. The calibration curve equations were carried out on the obtained data between the peak area and concentration, and their corresponding determination coefficients, analytical ranges, the LOD and LOQ are listed in Table 9. Excellent linearity was corroborated by the high value of coefficient $(\mathrm{R}>0.99)$ for four terpene trilactones. The precision, accuracy and stability of the analysis method were found to have acceptable RSD values, as shown in Table 10. All the RSD values were 
less than $5 \%$, which shows that the method had excellent precision values within the acceptable limits. Stability studies indicated that the sample was stable for $48 \mathrm{~h}$ when kept at $4 \pm 1{ }^{\circ} \mathrm{C}$. The average percent recoveries of the analytes at three different concentration levels from 80 to $120 \%$ of the sample concentration $(n=9)$ were between 93.29 and $103.14 \%$ with RSDs in the range of $2.1-3.67 \%$, which signifies a good accuracy of the developed method. These validation results indicates that the selected operating conditions used in the quantitative determination were appropriate.

Table 9 Linearity and analytical range

\begin{tabular}{cccccc}
\hline Component & Calibration curve equation & $\mathrm{r}$ & $\begin{array}{c}\text { Analytical } \\
\text { range }(\mu \mathrm{g} / \mathrm{mL})\end{array}$ & $\begin{array}{c}\text { LOD } \\
(\mathrm{ng} / \mathrm{mL})\end{array}$ & $\begin{array}{c}\text { LOQ } \\
(\mathrm{ng} / \mathrm{mL})\end{array}$ \\
\hline $\mathrm{BB}$ & $\mathrm{Y}=244176985.0580 \mathrm{x}+1959764.2083$ & 0.9935 & $4.813-77.000$ & 4.456 & 13.368 \\
$\mathrm{GC}$ & $\mathrm{Y}=339047410.0684 \mathrm{x}+329137.9917$ & 0.9931 & $0.552-8.832$ & 6.900 & 23.000 \\
$\mathrm{GA}$ & $\mathrm{Y}=261899741.6835 \mathrm{x}+313681.5000$ & 0.9962 & $1.024-16.384$ & 42.667 & 128.000 \\
$\mathrm{~GB}$ & $\mathrm{Y}=378664710.7361 \mathrm{x}+206893.8875$ & 0.9913 & $0.259-4.144$ & 3.238 & 10.792 \\
\hline
\end{tabular}

Table 10 Results of analytical precision, sample stability and accuracy experiments

\begin{tabular}{ccccccc}
\hline \multirow{2}{*}{ Component } & Injection & Intra-day & Inter-day & Sample & \multicolumn{2}{c}{ Recovery } \\
\cline { 6 - 7 } & precision & precision & precision & stability & Average value (\%) & RSD (\%) \\
& RSD (\%) & RSD (\%) & RSD (\%) & $(\%)$ & A & A \\
\hline BB & 0.71 & 2.62 & 3.82 & 0.84 & 93.29 & 2.10 \\
GC & 0.93 & 3.05 & 3.46 & 1.36 & 103.14 & 3.02 \\
GA & 1.87 & 3.00 & 2.99 & 1.25 & 102.73 & 2.96 \\
GB & 1.90 & 1.59 & 2.86 & 1.40 & 97.39 & 3.67 \\
\hline
\end{tabular}

\subsection{Application of the method}

The developed HPLC-MS method was applied to determine the four terpene trilactones of forty-five batches of EGBL from different manufacturers. The results are listed in Additional file 1: Table S3. The four terpene trilactones (BB, GC, GA, GB) 
contents of samples were $2.34 \%-4.68 \%, 0.81 \%-1.79 \%, 1.49 \%-3.58 \%, 0.85 \%-2.12 \%$, respectively. The sum of the four terpene trilactones was greater than $6 \%$ (Additional file 1: Table S3 and Fig. S5). As shown in Additional file 1: Fig.S5, BB is the major terpene trilactone in EGBL, followed by GA. Then twelve batches of those forty-five batches were compared with the HPLC-ELSD method results (data not shown). The paired $t$ test showed that the results of each compound and total terpene trilactones content determined by these two methods were not significant $(P>0.05)$, as shown in Table 11.

Table 11 Results of paired $t$ test of the two methods

\begin{tabular}{|c|c|c|c|c|c|c|c|c|}
\hline \multirow{3}{*}{$\begin{array}{l}\text { Components detected } \\
\text { by two methods }\end{array}$} & \multicolumn{5}{|c|}{ Paired differences } & \multirow{3}{*}{$t$} & \multirow{3}{*}{$\mathrm{df}$} & \multirow{3}{*}{ Sig.(two-tail) } \\
\hline & \multirow[t]{2}{*}{ Average } & \multirow{2}{*}{$\begin{array}{l}\text { Standard } \\
\text { deviation }\end{array}$} & \multirow{2}{*}{$\begin{array}{l}\text { Standard } \\
\text { error }\end{array}$} & \multicolumn{2}{|c|}{$\begin{array}{c}95 \% \text { confidence interval of } \\
\text { difference }\end{array}$} & & & \\
\hline & & & & Lower limit & Upper limit & & & \\
\hline BB-ELSD vs BB-MS & -0.1584 & 0.3442 & 0.0994 & -0.3771 & 0.0603 & -1.59 & 11 & 0.139 \\
\hline GC-ELSD vs GC-MS & -0.0423 & 0.0849 & 0.0245 & -0.0963 & 0.0116 & -1.73 & 11 & 0.112 \\
\hline GA-ELSD vs GA-MS & -0.0839 & 0.1775 & 0.0513 & -0.1967 & 0.0289 & -1.64 & 11 & 0.130 \\
\hline GB-ELSD vs GB-MS & 0.0386 & 0.0938 & 0.0271 & -0.0210 & 0.0983 & 1.43 & 11 & 0.181 \\
\hline $\begin{array}{l}\text { Total content-ELSD } \\
\text { vs total content-MS }\end{array}$ & -0.2460 & 0.6000 & 0.1730 & -0.6270 & 0.1350 & -1.42 & 11 & 0.183 \\
\hline
\end{tabular}

\section{Discussion}

The terpene trilactones are unique chemical compounds found exclusively in EGBL and related products and are therefore frequently used as marker components for quality control purposes [31]. For the determination of these compounds, HPLC-ELSD has remained the most widely used method for quantitative analysis of terpene trilactones. 
However, the HPLC-ELSD method repeatedly requires liquid-liquid extractions using ethyl acetate, leading to tedious process, high solvent consumption and long processing time [32]. In this study, a sensitive and robust HPLC-MS was developed to simply and rapidly measure the terpene trilactones in EGBL. Compared with the method of HPLC-ELSD, the developed HPLC-MS analysis method had many advantages, such as simple sample pretreatment, low solvent consumption, small sample weight and short pretreatment time.

$\mathrm{AQbD}$ is an advanced quality control approach that has been gradually implemented in the optimization of analytical methods for Chinese medicine [33]. It was observed that the AQbD concept could not only help deepen understanding of the analysis process but also improve the analytical quality for terpene trilactones of EGBL. From Additional file 1: Fig. S1, 2, 3, and 4, contour plots exhibit an easy, quick and an impressive way of interpreting the relationship between the CMPs and CMAs. High flow rate of mobile phase would lead to a low response for all the CMAs, which may be due to the fact that more analyte ions are detected at a certain scan dwell time when the flow rate of mobile phase is lower. Both gas flow rate and gas temperature have positive influence on all the CMAs for the range of values tested in the study, which is due to the fact that higher gas flow rate and gas temperature are beneficial for the desolvation process of the electrosprayed droplets, the formation and control of the size of these droplets. Furthermore, the hypercube design space was developed, which is considered as a "safe zone" where no considerable changes in the CMAs are observed as a 
consequence of small, deliberate changes of CMPs. The CMPs ranges, that marked the hypercube design space and minimum responses requirements, together formed the analytical control strategy, which can ensure the desired analytical quality for terpene trilactones in EGBL. This analytical control strategy is promising for application to methods development for other Chinese medicines.

In addition, with the growth of the demand for EGBL, many countries have become the main countries for the production of EGBL, especially China. Therefore, a robust and simple analysis method for terpene trilactones in EGBL and related products is necessary. Thus, the developed HPLC-MS is an alternative method for the determination of terpene trilactones in commercial EGBL and related products, leading to more robust and effective.

\section{Conclusion}

Benefits of employing AQbD approach during the development of analytical method have been illustrated in the case study presented here. The current studies highlight a novel AQbD to develop a rapid, sensitive and robust LC-MS method for the determination of terpene trilactone contents of EGBL. The developed method is robust and reliable for analysis over the method operational hypercube design space. Risk assessment was applied in this work, Plackett-Burman design experiment was implemented for selecting the CMPs. Four CMPs of the flow rate of mobile phase, the proportion of formic acid in the mobile phase, gas flow rate and gas temperature were found with a standard partial regression coefficient method. The obtained mathematical 
models allowed a good understanding of the CMPs effects on the CMAs, employing the Box-Behnken designed experiment. With respect to the aforementioned CMAs, the design space was computed using a Monte-Carlo simulation method. On basis of hypercube design space, the analytical control strategy was established demanding strict controls on the CMPs. Then the developed method was validated and utilized for the analysis of terpene trilactones of EGBL. Compared with the reported HPLC-ELSD methods with complicated sample preparation and long analysis time, the developed HPLC-MS method leads to more effective due to simple preparation and shorter analysis time, which is an alternative method for the determination of terpene trilactones in commercial EGBLs.

\begin{abstract}
Abbreviations
EGBL: Ginkgo biloba leaf extract; AQbD: analytical quality by design; ATP: analytical target profile; CMAs: critical method attributes; CMPs: critical method parameters; BBD: Box-Behnken design; GA: ginkgolides A; GB: ginkgolides B; GC: ginkgolides C; BB: bilobalide; QbD: Quality by Design; CQAs: critical quality attributes; LOD: limit of detection; LOQ: limit of quantification; SIM: selected ion monitoring.
\end{abstract}

\title{
Acknowledgements
}

Not applicable.

\section{Authors' contributions}

HQ and SZ designed the study. SZ conducted the study and drafted the manuscript. 
XG and HQ supervised the study, and HQ revised the manuscript. XG, LW and XW provided the technical support and advices for the study. All authors read and approved the final manuscript.

\section{Funding}

This work was supported by the National Science \& Technology Major Project of China (No. 2018ZX09201011-002).

\section{Availability of data and materials}

The research data generated from this study are included in this article and its supplementary information files.

\section{Ethics approval and consent to participate}

Not applicable.

\section{Consent for publication}

Not applicable.

\section{Competing interests}

The authors declare that they have no competing interests.

\section{Author details}

Pharmaceutical Informatics Institute, College of Pharmaceutical Sciences, Zhejiang University, Hangzhou 310058, China.

\section{Reference}

1. Li YJ, Zhang CF, Huang WZ, Geng T, Wang ZZ, Zhao BJ, et al. Simultaneous determination of ginkgolides $\mathrm{A}, \mathrm{B}$ and $\mathrm{K}$ in human plasma by UPLC-MS/MS and 
its application to the pharmacokinetic study of Ginkgo Diterpene Lactone Meglumine Injection in humans. Anal Methods. 2016;8(11):2341-48.

2. Singh B, Kaur P, Singh, RD, Ahuja PS. Biology and chemistry of Ginkgo biloba. Fitoterapia. 2008;79(6): 401-18.

3. Johnson RT, Lunte CE. A capillary electrophoresis electrospray ionization-mass spectrometry method using a borate background electrolyte for the fingerprinting analysis of flavonoids in Ginkgo biloba herbal supplements. Anal Methods. 2016;8(16):3325-32.

4. Shi CA, Liu JJ, Wu FM, Yew DT. Ginkgo biloba extract in Alzheimer's disease: from action mechanisms to medical practice. Int J Mol Sci. 2010;11(1):107-23.

5. Roland P, Nergård C. Ginkgo biloba-effekt, bivirkninger og interaksjoner. Tidsskr Nor Legeforen. 2012;132:956-9.

6. Zhou W, Chai H, Lin P H, Lumsden AB, Yao Q, Chen CY. Clinical use and molecular mechanisms of action of extract of Ginkgo biloba leaves in cardiovascular diseases. Cardiovasc Drug Rev. 2006;22(4):309-19.

7. Unger M. Pharmacokinetic drug interactions involving Ginkgo biloba. Drug Metab Rev. 2013;45(3):353-85.

8. Shu PH, Sun MY, Li JP, Zhang LX, Xu HC, Lou YY, et al. Chemical constituents from Ginkgo biloba leaves and their cytotoxicity activity. J Nat Med. 2020;74(1):269-74.

9. Xiong XJ, Liu WY, Yang XC, Feng B, Zhang Y, Li SJ, et al. Ginkgo biloba extract 
for essential hypertension: a systemic review. Phytomedicine. 2014;21(10):1131-6.

10. Braga LR, Rosa AA, Dias ACB. Synthesis and characterization of molecularly imprinted silica mediated by Al for solid phase extraction of quercetin in Ginkgo biloba L. Anal Methods. 2014;6(12): 4029-37.

11. Haruyama T, Nagata K. Anti-influenza virus activity of Ginkgo biloba leaf extracts. J Nat Med. 2013;67(3):636-42.

12. Jensen AG, Ndjoko K, Wolfender JL, Hostettmann K, Camponovo F, Soldati F. Liquid chromatography-Atmospheric pressure chemical ionisation/Mass spectrometry: a rapid and selective method for the quantitative determination of ginkgolides and bilobalide in ginkgo leaf extracts and phytopharmaceuticals. Phytochem Analysis. 2002;13(1):31-8.

13. Lobstein-Guth A, Briançon-Scheid F, Anton R. Analysis of terpenes from Ginkgo biloba L. by high-performance liquid chromatography. J Chromatogr A. $1983 ; 267: 431-8$.

14. Pietta P, Mauri P, Rava A. Analysis of terpenes from Ginkgo biloba L. extracts by reversed phase high-performance liquid chromatography. Chromatographia. 1990;29(5):251-3.

15. Camponovo FF, Wolfender JL, Maillard MP, Potterat O, Hostettmann K. Evaporative light scattering and thermospray mass spectrometry: two alternative methods for detection and quantitative liquid chromatographic determination of ginkgolides and bilobalide in Ginkgo biloba leaf extracts and 
phytopharmaceuticals. Phytochem Analysis. 1995;6(3):141-8.

16. Chen $\mathrm{P}, \mathrm{Su} \mathrm{XL}$, Nie LH, Yao SZ, Zeng JG. Analysis of ginkgolides and bilobalides in Ginkgo biloba L. extract for its production process control by high-performance liquid chromatography. J Chromatogr Sci. 1998;36(4):197-200.

17. Van Beek TA, Montoro P. Chemical analysis and quality control of Ginkgo biloba leaves, extracts, and phytopharmaceuticals. J Chromatogr A. 2009;1216(11):2002-32.

18. Carrier DJ, Chauret N, Mancini M, Coulombe P, Neufeld RJ, Weber ME, et al. Detection of ginkgolide A in Ginkgo biloba cell cultures. Plant Cell Rep. 1991;10(5):256-9.

19. Lang QY, Yak HK., Wai CM. Selective dissolution and one step separation of terpene trilactones in ginkgo leaf extracts for GC-FID determination. Talanta. 2001;54(4)673-80.

20. Liang TF, Miyakawa T, Yang JW, Ishikawa T, Tanokura M. Quantification of terpene trilactones in Ginkgo biloba with a ${ }^{1} \mathrm{H}$ NMR method. J Nat Med. 2018;72(3):793-7.

21. López-Gutiérrez N, Romero-González R, Vidal JLM, Frenich AG. Quality control evaluation of nutraceutical products from Ginkgo biloba using liquid chromatography coupled to high resolution mass spectrometry. J Pharmaceut Biomed Anal. 2016;121:151-60.

22. Iliou K, Malenovic A, Loukas YL, Dotsikas Y. Analysis of potential genotoxic 
impurities in rabeprazole active pharmaceutical ingredient via liquid chromatography-tandem mass spectrometry, following quality-by-design principles for method development. J Pharm Biomed Anal. 2018;149,410-8.

23. Hashem H, El-Sayed HM. Quality by design approach for development and validation of a RP-HPLC method for simultaneous determination of co-administered levetiracetam and pyridoxine $\mathrm{HCl}$ in prepared tablets. Microchem J. 2018;143:55-63.

24. Debrus B, Lebrun P, Kindenge JM, Lecomte F, Ceccato A, Caliaro G. Innovative high-performance liquid chromatography method development for the screening of 19 antimalarial drugs based on a generic approach, using design of experiments, independent component analysis and design space. $\mathrm{J}$ Chromatogr A. 2011;1218(31):5205-15.

25. Wang L, Qu HB. Development and optimization of SPE-HPLC-UV/ELSD for simultaneous determination of nine bioactive components in Shenqi Fuzheng Injection based on Quality by Design principles. Anal Bioanal Chem. 2016;408(8):2133-45.

26. Kostic N, Dotsikas Y, Malenovic A, Stojanovic BJ, Rakic T, Ivanovic D, et al. Stepwise optimization approach for improving LC-MS/MS analysis of zwitterionic antiepileptic drugs with implementation of experimental design. J Mass Spectrom. 2013;48(7):875-84.

27. Kochling J, Wu W, Hua YM, Guan Q, Castaneda-Merced J. A platform analytical 
quality by design (AQbD) approach for multiple UHPLC-UV and UHPLC-MS methods development for protein analysis. J Pharm Biomed Anal. 2016 125:130-9.

28. Rozet E, Lebrun P, Hubert P, Debrus B, Boulanger B. Design spaces for analytical methods. Trac-Rend Anal Chem. 2013;42:157-67.

29. Shao JY, Cao W, Qu HB, Pan JY, Gong XC. A novel quality by design approach for developing an HPLC method to analyze herbal extracts: A case study of sugar content analysis. Plos One. 2018;13(6): e0198515.

30. Ferreira SLC, Caires AO, Borges TDS, Lima AMS, Silva LOB, Santos WNLD. Robustness evaluation in analytical methods optimized using experimental designs. Microchem J. 2017;131:163-9.

31. Ren C, Ji YQ, Liu H, Wang Z, Wang JH, Zhang CY, et al. Effects of Ginkgo biloba extract EGb761 on neural differentiation of stem cells offer new hope for neurological disease treatment. Neural Regen Res. 2019;14(7):1152-7.

32. Dubber MJ, Kanfer I. Determination of terpene trilactones in Ginkgo biloba solid oral dosage forms using HPLC with evaporative light scattering detection. Pharm Biomed Anal. 2006; 41(1):135-40.

33. Dai SY, Xu B, Zhang Y, Li JY, Sun F, Shi XY, et al. Establishment and reliability evaluation of the design space for HPLC analysis of six alkaloids in Coptis chinensis (Huanglian) using Bayesian approach. Chin J Nat Med. 2016;14(9):697-708. 


\section{Figures}

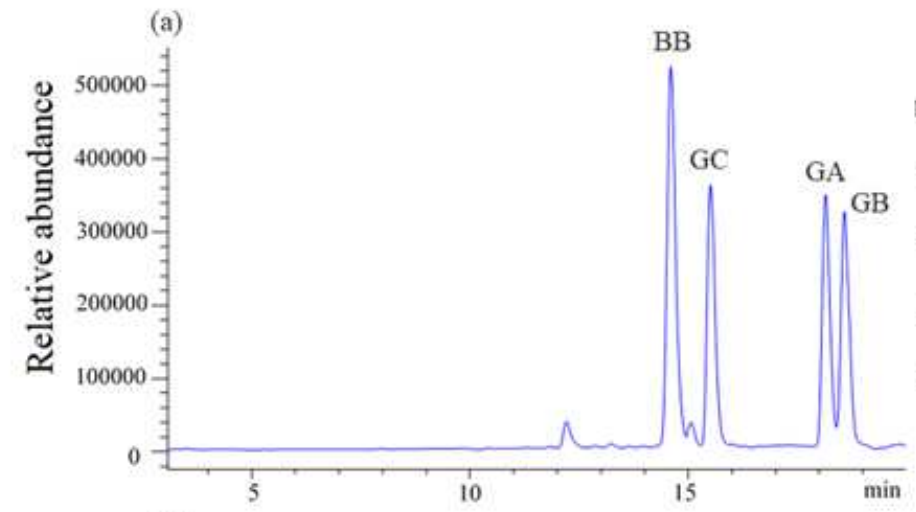

(c)
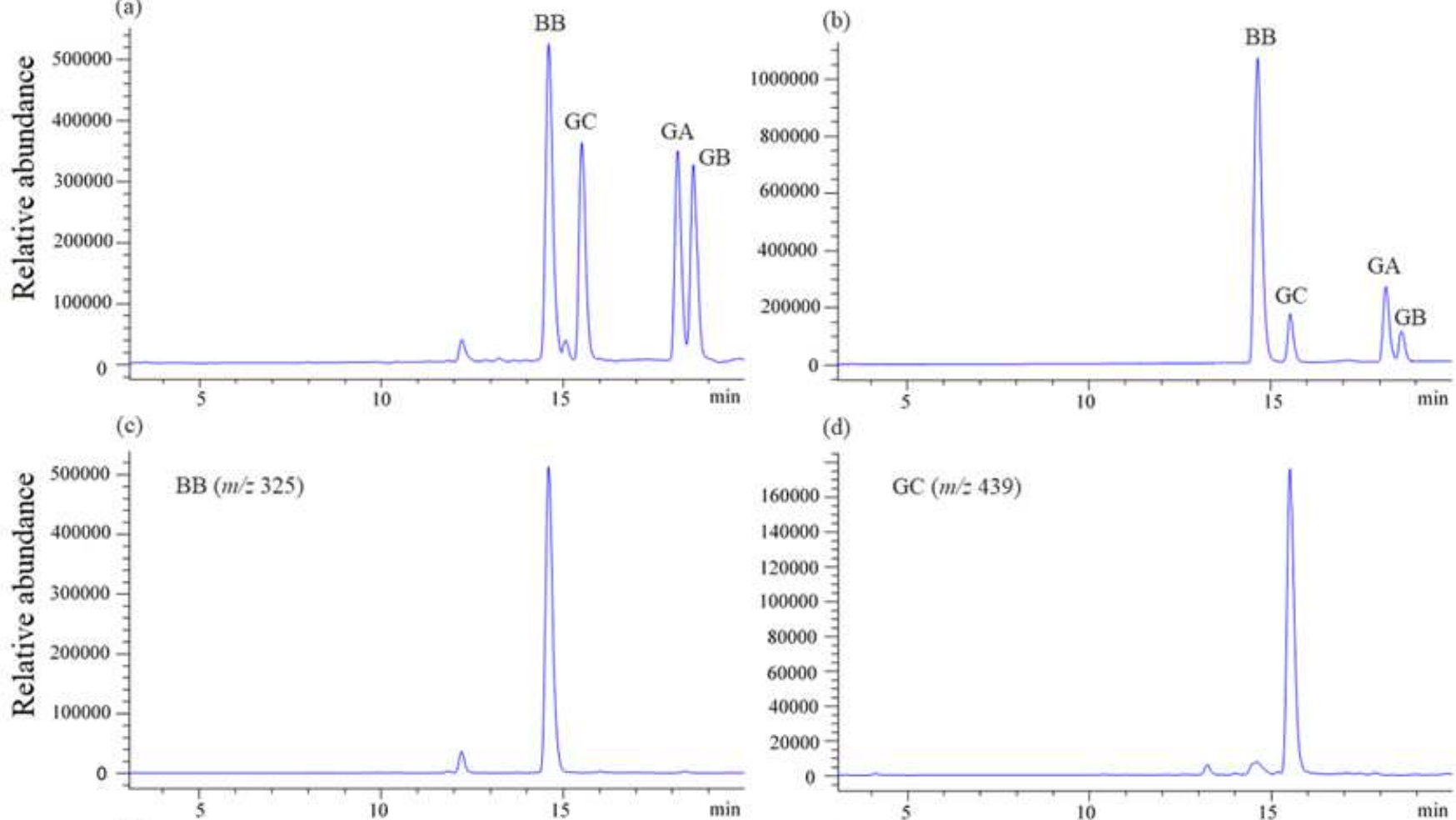

(d)

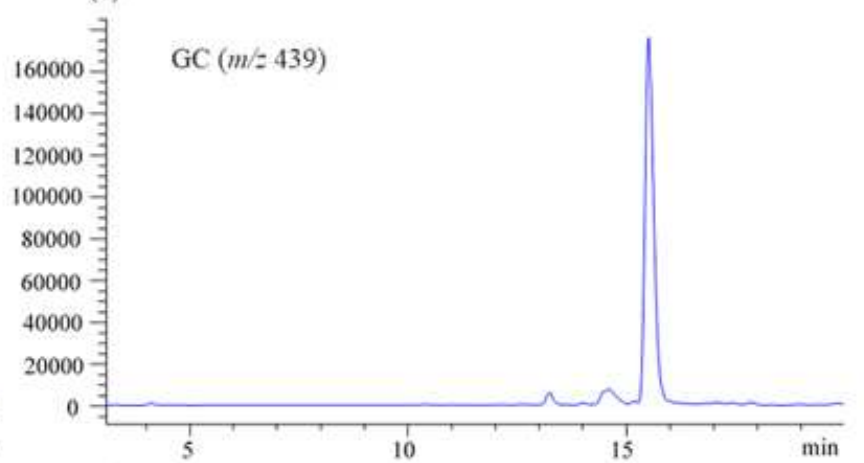

(e)

(f)
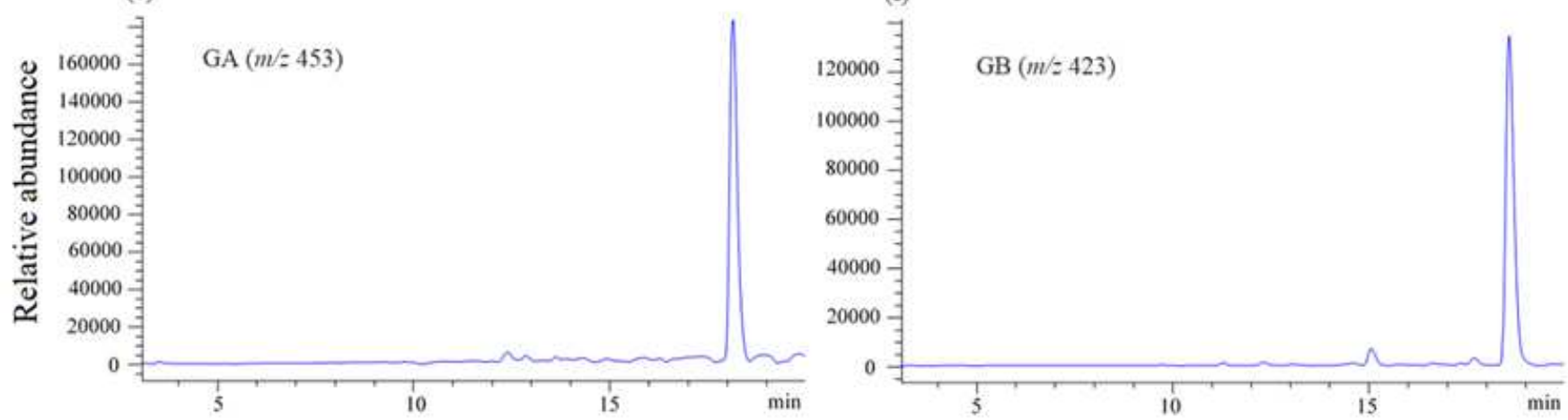

Figure 1

Typical selected ion monitoring (SIM) chromatograms of EGBL and standard solution. (a-b) All terpene trilactones ions traces for EGBL and standard solution, respectively; (c-f) The SIM spectra of BB, GC, GA, GB for EGBL, respectively 


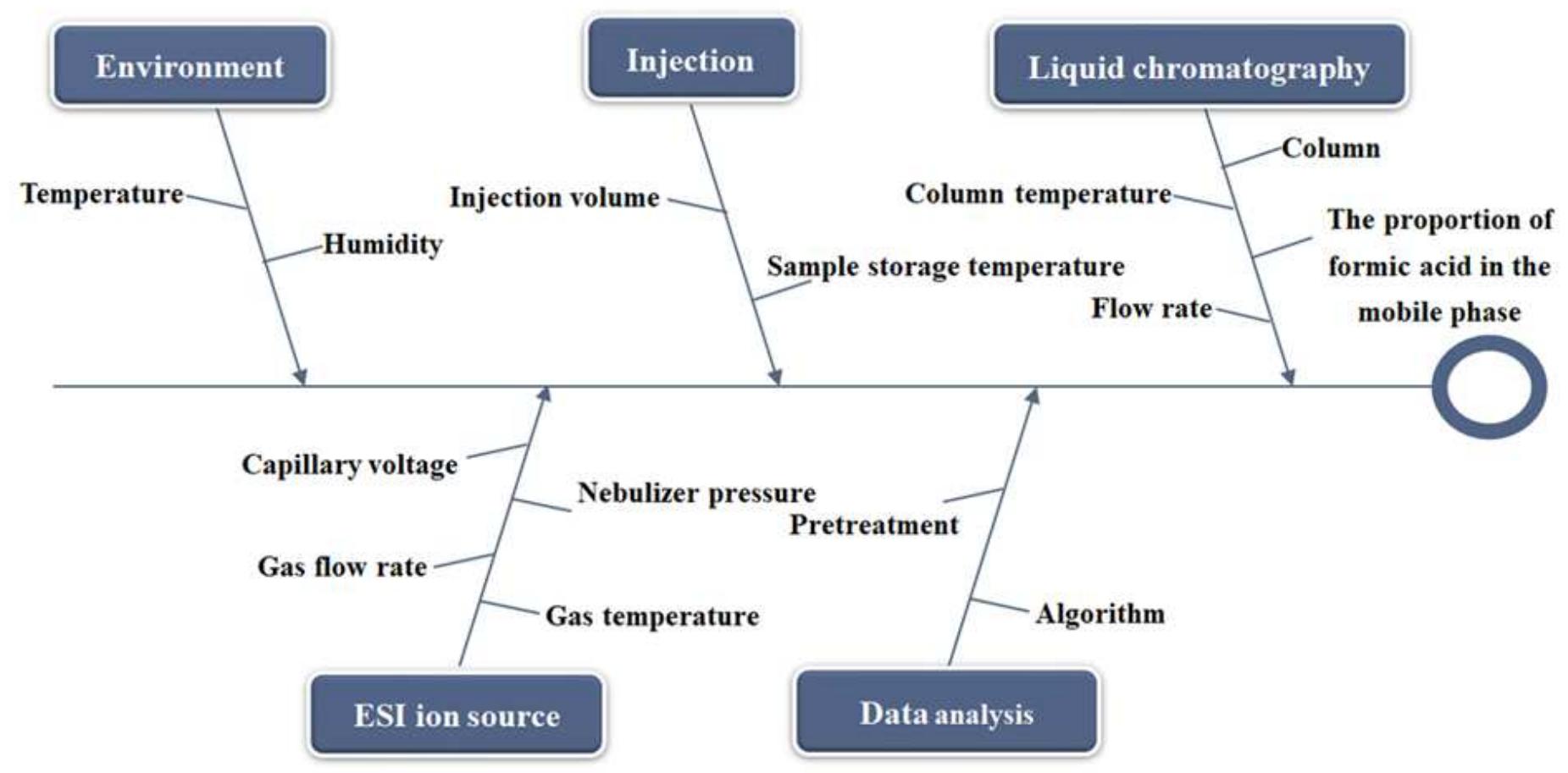

Figure 2

Ishikawa diagram for potential CMPs selection
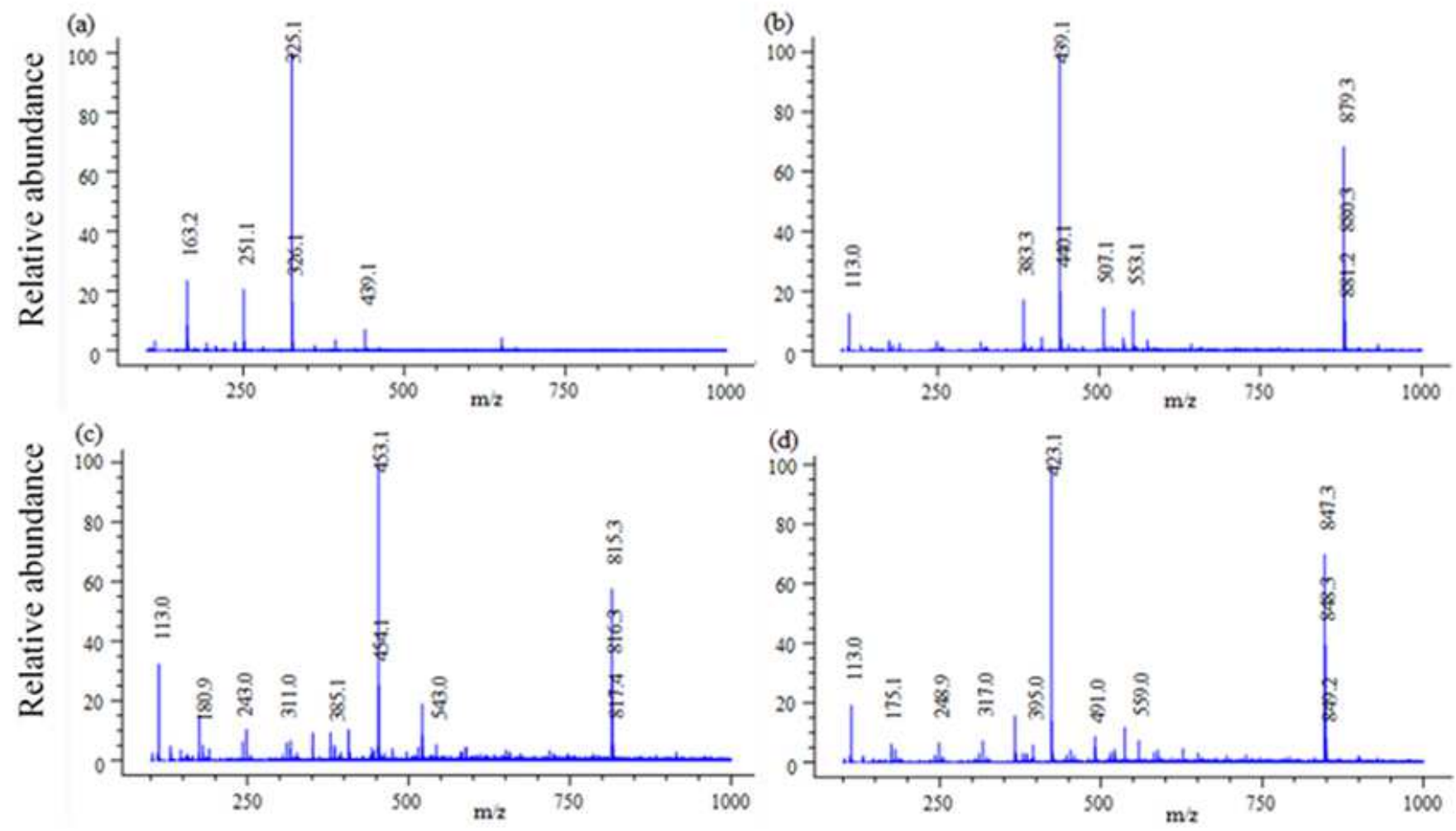

Figure 3

Full scan mass spectrum of (a) Bilobalide; (b) Ginkgolide C; (c) Ginkgolide A; (d) Ginkgolide B. 
(a)

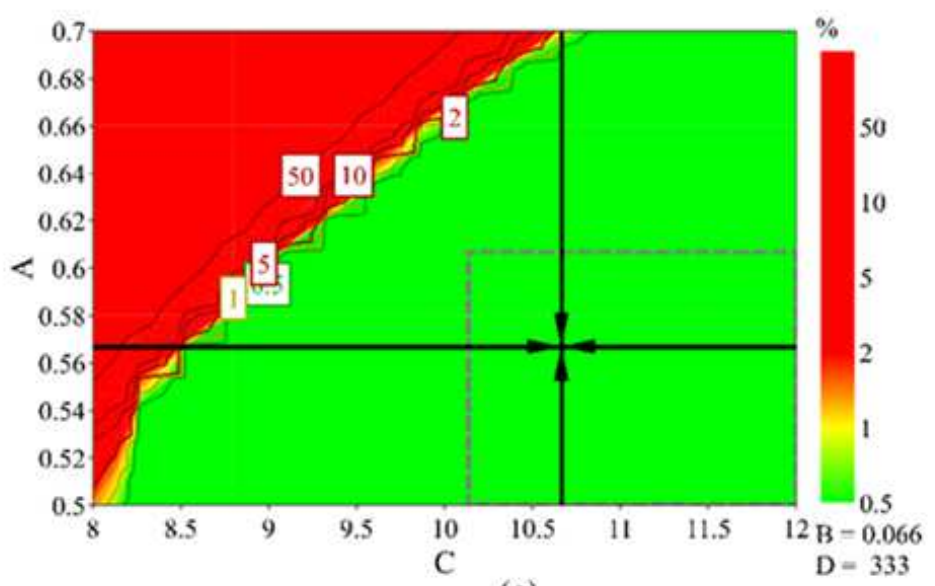

(b)

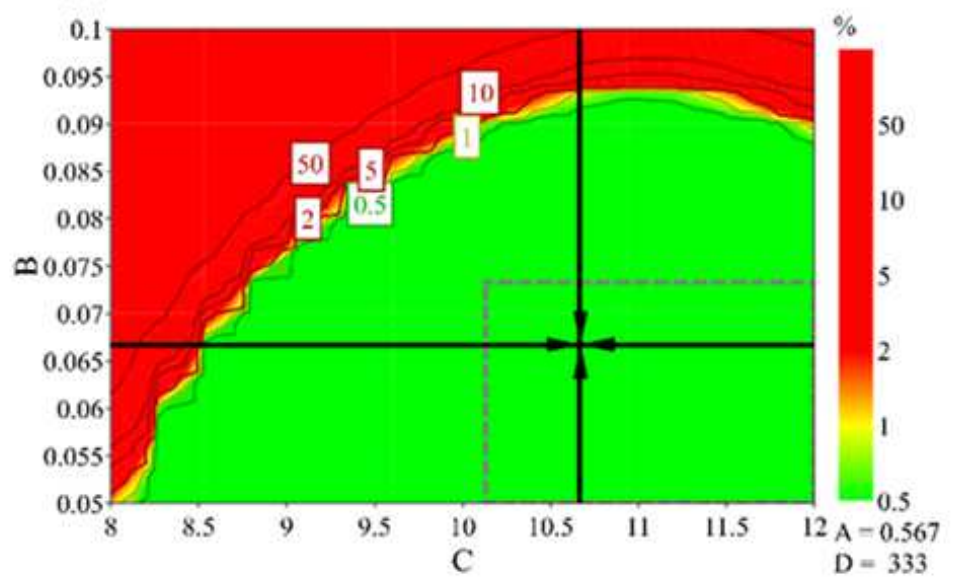

(c)

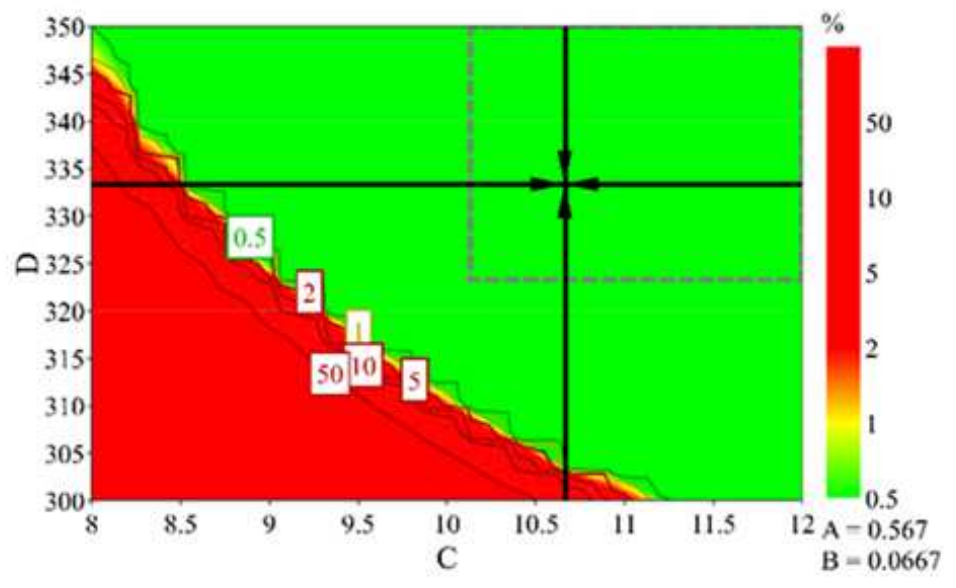

Figure 4

Design space plot with a robust setpoint. A to $D$ represent flow rate of mobile phase, the proportion of formic acid in the mobile phase, gas flow rate and gas temperature, respectively. (Color bar refers to the risk of error to attain CMA goals). The largest hypercube design space is plotted as a gray dashed frame with a probability of failure of less than $1 \%$. The robust setpoint is displayed as arrow crossing.

\section{Supplementary Files}

This is a list of supplementary files associated with this preprint. Click to download.

- Additionalfile1.docx

- Additionalfile1.docx

- Additionalfile1.docx 